\title{
Estimating Solar Forcing of Climate Change during the Maunder Minimum
}

\author{
By JUDITH LEA N ${ }^{1}$, ANDREW SKUMANICH \\ ORAN R. WHITE ${ }^{2}$ AND DAVID RIND \\ ${ }^{1}$ Naval Research Laboratory, Washington DC, 20375, USA \\ ${ }^{2}$ High Altitude Observatory, National Center for Atmospheric Research, \\ Boulder, CO 80303, USA \\ ${ }^{3}$ Goddard Institute for Space Studies, New York, NY 10025, USA
}

Solar variability models which account for contemporary irradiance variations in terms of modulation by dark sunspots and bright faculae on the solar disk are used to estimate solar radiative output during the Maunder Minimum by postulating the absence of such sources, and additional reduction in the basal solar emission. When the resultant irradiance reduction of $0.25 \%$ relative to contemporary mean levels is input to the GISS GCM, the global mean temperature is reduced by $0.46^{\circ} \mathrm{C}$. However, as a result of differential heating of the land and oceans, some regions may $\mathrm{cool}$, and others warm, by as much $1^{\circ} \mathrm{C}$, in response to the reduced solar irradiance.

\section{Introduction}

Evidence for variations in the activity level of the Sun, illustrated by the irradiance and sunspot data in Figure 1, exists on time scales from days to centuries, and raises the possibility of solar forcing of past and future climate change. During the period from about 1645 to 1715 , called the Maunder Minimum, sunspots were absent from the solar disk for long periods (Eddy 1976) whereas numerous sunspots occur at contemporary activity maxima, and are rarely absent even at activity minima. The Maunder Minimum in the sunspot record is but the most recent of recurrent epochs of depressed solar activity evident in both the ${ }^{10} \mathrm{Be}$ and ${ }^{14} \mathrm{C}$ cosmogenic solar activity proxies (McHargue \& Damon 1991). Since the Maunder Minimum, solar activity has risen steadily to the current Modern Maximum and, according to the ${ }^{10} \mathrm{Be}$ proxy data, has now reached levels as high as those last seen in the 12 th century. Space-based measurements with sufficient precision to identify real changes in solar radiative have been made near the peak of the Modern Maximum but for barely more than one 11-year solar activity cycle (Willson \& Hudson 1991; Hoyt et al. 1992).

The variations in solar radiative output recorded by the space-based monitoring are associated with eruptions of magnetic activity into the solar atmosphere, producing regions where the local solar emission may be depleted (in sunspots) or enhanced (in faculae). As the mix of the bright and dark active regions changes throughout the 11-year activity cycle, so too does the integrated radiative output from the solar disk (e.g., Foukal \& Lean 1988). Numerical estimates of solar radiative output during times beyond the limited observational data base are possible using variability models in which the relative roles of sunspot blocking and faculae enhancement are derived from the extant radiometry. With this approach we investigate the effect of various scenarios of depleted bright magnetic elements on the full disk solar emission, estimate the probable reduction of the total solar irradiance during the Maunder Minimum, and use the Goddard Institute for Space Studies (GISS) General Circulation Model (GCM) to calculate the resultant geographical temperature changes that might be expected from this reduction. 


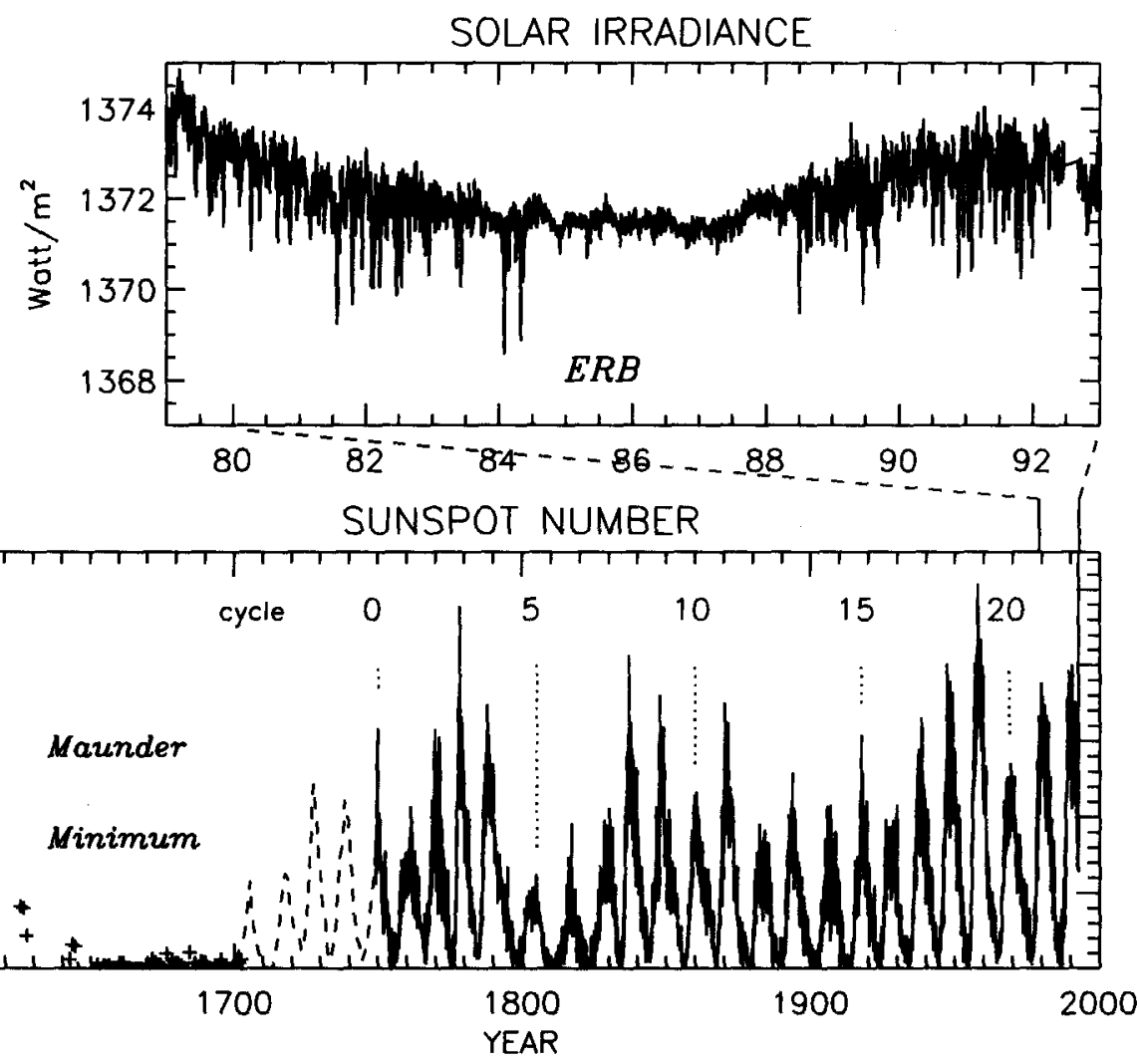

FIgURE 1. Changes in solar activity over daily, decadal and century time scales, as evidenced in (upper panel) total solar radiative output (Hoyt et al. 1992) and (lower panel) the sunspot number.

\section{Solar irradiance observations and models}

Satellite radiometry during the past decade has established that the solar radiative output varies continuously, on all time scales and at all wavelengths measured thus far (Lean 1991). Shown in Figure 2 are variations during solar cycles 21 and 22 in the total solar irradiance, S, measured by the ACRIM radiometer (Willson \& Hudson 1991) and in the ultraviolet spectral irradiance of the H I Lyman $\alpha$ line at $121.57 \mathrm{~nm}$, measured by the Solar Mesosphere Explorer (Rottman 1988). Reductions of as much as $0.3 \%$ in total solar irradiance can occur during the Sun's 27-day rotation as sunspots, which block solar radiation, rotate across the face of the Sun's disk seen at the Earth. Over longer time scales, from the minimum to the maximum of the 11-year solar cycle, an irradiance increase of $0.1 \%$ results from the build-up of enhanced emission in bright regions, which more than compensates for the reduction in the dark sunspots. Solar variability at ultraviolet wavelengths exceeds that of the total irradiance. Variations over the solar cycle are of the order of 5-10\% at $200 \mathrm{~nm}$ and $50 \%$ at H I Lyman $\alpha$ (Rottman 1988; Lean 1991). In the spectral region 120 to $250 \mathrm{~nm}$, the variations exhibit very similar temporal structure (Donnelly 1988), responding alike to the presence on the solar disk of bright magnetic regions, the dominant source of variability over both the 27-day and 11-year 

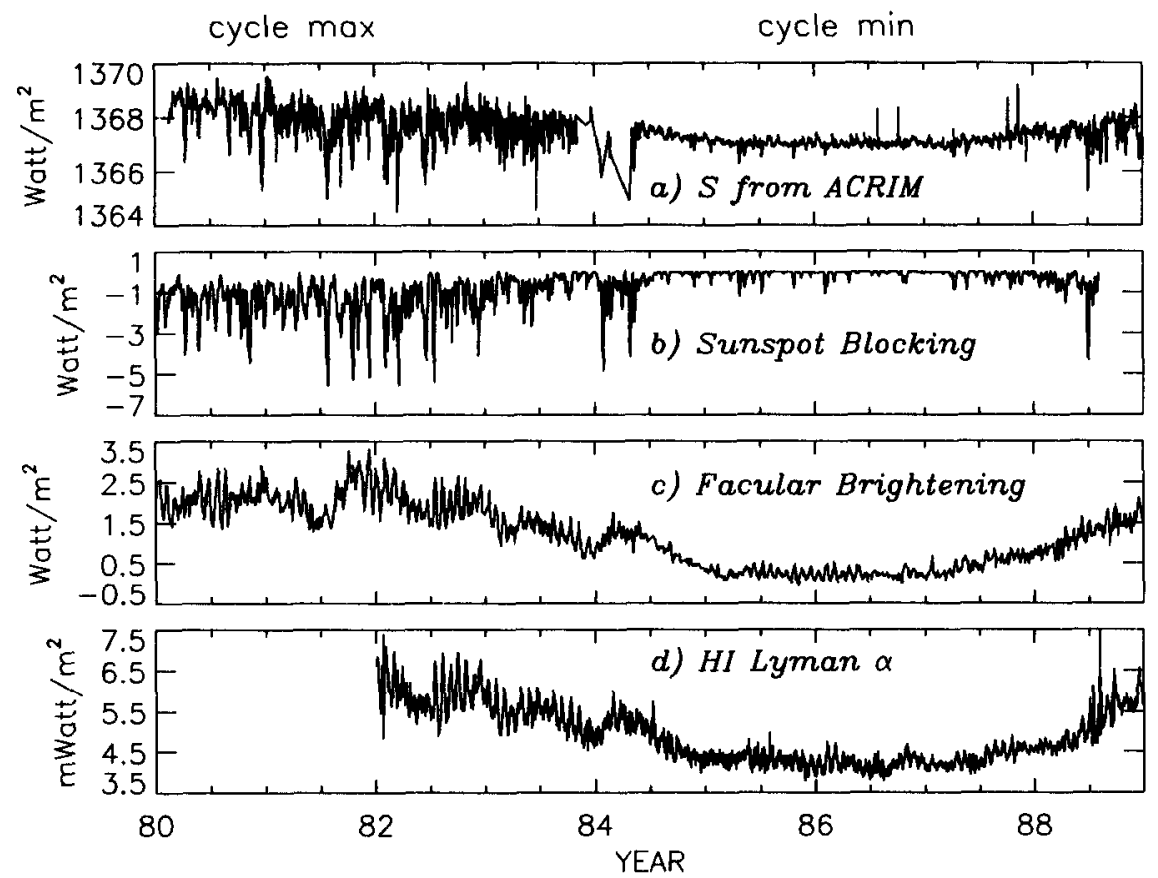

Figure 2. Variations throughout the 11-year solar activity cycle in a) total solar radiative ouput, b) sunspot blocking, c) facular brightening and d) the UV flux at HI Lyman $\alpha$.

cycles. In comparison, the total solar radiative output has a different temporal structure that reflects the competing effects of both bright faculae and dark sunspots.

Also shown in Figure 2 are the variations in the sunspot blocking and the facular brightening associated with magnetic modulation of the quiet Sun irradiance, $S_{Q}$, during solar cycles 21 and 22 . These quantities parameterize, respectively, the dark and bright regions whose competing effects determine the overall solar radiative output. The sunspot blocking was calculated according to Foukal (1981). The facular brightening was estimated from ground-based measurements of solar Ca II K emission, linearly regressed against $S-S_{Q}\left(1+P_{s}\right)$ (Livingston et al. 1988), where $S_{Q} P_{s}$ is a measure of the sunspot blocking. As expected, and evident in Figure 2, the H I Lyman $\alpha$ variations, which reflect changes in enhanced emission from bright faculae, track closely the calculations of facular brightening derived from Ca II K data (White et al. 1990), as well as determined directly from the ACRIM radiometry (Foukal \& Lean 1990).

Models of solar irradiance variability constructed from the ground-based sunspot blocking and facular brightening time series in Figure 2 are generally able to reproduce both the 27-day and 11-year variations measured by the satellite radiometers during the past decade (Foukal \& Lean 1990; Willson \& Hudson 1991; Lean 1991). However, the radiometric database extends over barely one 11-year solar activity cycle and some discrepancies between the models and measurements remain unexplained (Foukal \& Lean 1988; Kuhn \& Libbrecht 1991; Willson \& Hudson, 1991). Variations that may be occurring on time scales longer than the 11-year cycle have yet to be recorded by the radiometers, 
or included in these types of variability models (Fröhlich 1994). As well, refinements to the sunspot blocking function are in progress, incorporating the dependence of sunspot contrast on spot area (Brandt et al. 1992).

\section{Solar and stellar CA II flux observations}

The generally good agreement between the contemporary solar irradiance measurements and reconstructions from sunspot blocking and facular brightening time series provides strong evidence that variations in total solar irradiance arise from magnetic modulation of a quiet signal, at least for the recent 11-year solar cycle. With knowledge of the character and evolution of solar magnetic active regions, inferences can thus be made about irradiance variations on longer time scales. Observations of the Sun and stars made at the wavelength of ionized calcium have proven particularly helpful in this regard because $\mathrm{Ca}$ II emission is enhanced in magnetic active regions. On the Sun, these regions of bright $\mathrm{Ca}$ emission are spatially coherent with bright photospheric faculae, and thus provide proxy data for the brightness source of irradiance variations.

Spatially resolved observations of the solar disk in the $\mathrm{K}$ Fraunhofer line of Ca II indicate that large active regions are a significant component of disk-integrated brightness variations. However, studies comparing resolved $\mathrm{Ca}$ II $\mathrm{K}$ images with independent measurements of the disk-integrated flux indicate that the large active regions are not the only component. To explain the 11-year cycle increase in the disk-integrated Ca II $\mathrm{K}$ flux data, Skumanich et al. (1984) postulated that in addition to increased emission from large active regions, the area of the solar disk covered by chromospheric network was larger at solar maximum than the $39 \%$ coverage determined by Skumanich et al. (1975) for the quiet Sun. This additional brightness source, postulated to arise in active network, may explain why the bolometric facular brightening is sufficient to exceed the sunspot blocking over the 11-year activity cycle, giving rise to total radiative output variations that are in phase with solar magnetic activity. Global variations in solar structure, rather than magnetic activity alone, may also provide an explanation for this (Kuhn \& Libbrecht 1991).

A broader perspective of the range of temporal variability possible for the Sun is afforded from comparative observations of solar-like stars (Lockwood 1994). This is illustrated in Figure 3 which compares contemporary solar $\mathrm{Ca}$ II emission with that from 13 solar-like stars. Values for both the solar Ca II K index and the stellar Ca II HK index are indicated, using the conversion $\mathrm{HK}=0.04+1.53^{\circ} \mathrm{K}$ (White et al. 1992).

The Sun's $\mathrm{Ca} \mathrm{K}$ emission is seen to be typical of Ca emission levels from the brightest one-third of those solar-like stars that exhibit cyclic patterns similar to the Sun's 11-year cycle ( $\mathrm{HK}>0.15)$. This is consistent with the contemporary solar Ca II data having been collected during an epoch in which overall levels are higher than in the past few hundred years, as indicated by the sunspot and cosmogenic isotope data. In stars for which long-term cycles are absent ( $\mathrm{HK}<0.15)$, the Ca II emission is reduced even below the minimum levels seen in the cycling stars. One explanation for the reduced $\mathrm{Ca}$ emission in some solar-like stars is that the fraction of their disk covered by bright Ca emission is less than the $39 \%$ coverage of the contemporary minimum Sun. White et al. (1992) have demonstrated that this hypothesis can explain the range of $\mathrm{Ca}$ II emission in the cycling stars (the upper portion of the stellar distribution in Figure 3). However, reduction of the basal solar emission (i.e., the emission from the centers of the chromospheric cells around which the active network forms) to a value as low as that emitted from only $15 \%$ of the contemporary Sun must also be invoked to account for Ca II emission levels of the non-cycling stars (the lower part of the distribution in Figure 3). 


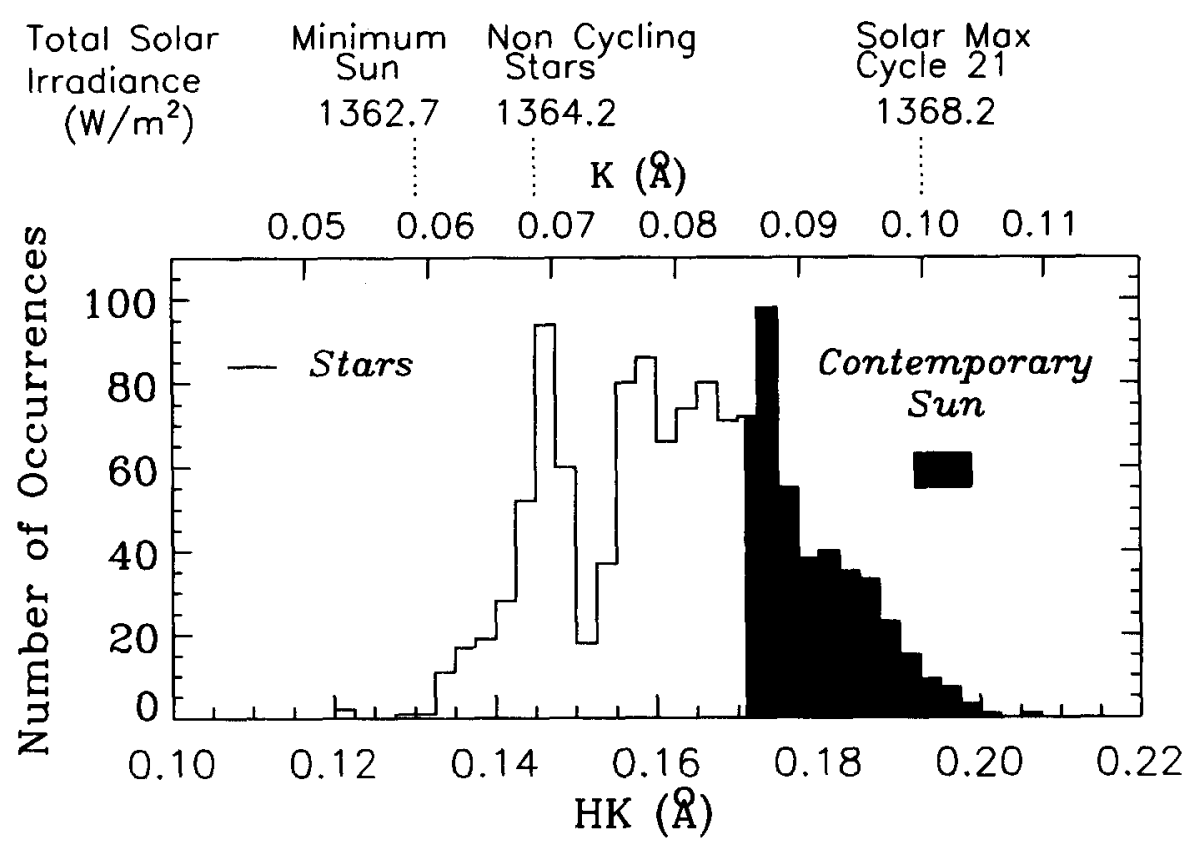

Figure 3. Distribution of Ca emission from 13 solar-like stars, compared with the emission from the contemporary Sun. Stars with HK >0.15 exhibit cycles similar to the 11-year solar activity cycle.

\section{Inferences for the Maunder Minimum Sun}

During the Maunder Minimum, the absence of sunspots (Eddy 1976), indicates zero sunspot blocking, $P_{s}=0$. The level of facular brightening can be inferred from solar and stellar $\mathrm{Ca}$ emission data. Assuming that the distribution of solar-like stars in Figure 3 is pertinent to the Sun, Baliunas \& Jastrow (1990) postulated that the non-cycling stars $(\mathrm{HK}<0.15)$ are in phases of inactivity analogous to the Maunder Minimum Sun. In this case, the solar Ca II K level for the Maunder Minimum is 0.0686 , the peak of the distribution of the non-cycling stars. This value is significantly less than the lowest level of $\mathrm{K}=0.085$ seen in the contemporary minimum Sun.

The level of solar facular emission corresponding to the Maunder Minimum Ca II K value is obtained from the correlation of contemporary $\mathrm{Ca}$ II $\mathrm{K}$ flux with direct solar radiometery, as indicated in Figure 4 . The values of $\mathrm{S}-\mathrm{S}_{\mathrm{Q}}\left(1+\mathrm{P}_{\mathrm{s}}\right)$ were calculated using $\mathrm{S}$ from the ACRIM radiometry, with $\mathrm{S}_{\mathrm{Q}}=1366.9 \mathrm{Watt} / \mathrm{m}^{2}$ and $\mathrm{P}_{\mathrm{s}}$ determined from ground-based sunspot data following Foukal (1981), using a mean spot contrast of 0.67 . From Figure 4, a Ca II K value of 0.0686 (non-cycling stars) implies a reduction in facular emission of $2.7 \mathrm{Watt} / \mathrm{m}^{2}$ relative to contemporary solar minimum values. Thus, the results in Figure 4 imply that the total solar irradiance was reduced to $1364.2 \mathrm{Watt} / \mathrm{m}^{2}$ during the Maunder Minimum.

\section{Solar forcing of climate during the Maunder Minimum}

The Maunder Minimum coincided with the coldest period of the Little Ice Age. Since then, the mean global temperature of the Earth has increased by about $1^{\circ} \mathrm{C}$, although 


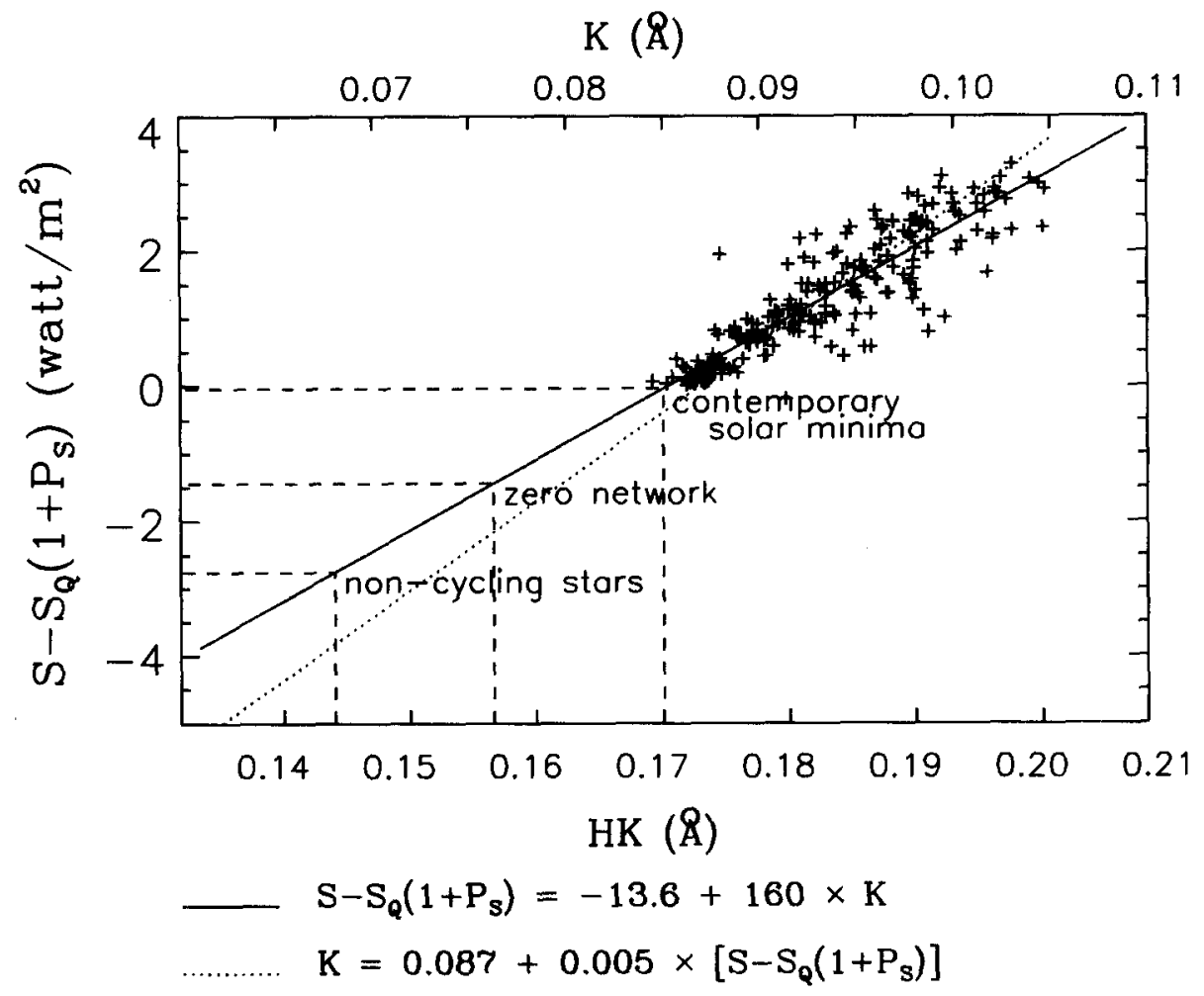

Figure 4. Correlation between solar Ca emission (abscissa) and total irradiance brightness (ordinate), from Lean et al. (1992). The dotted line is the fit obtained when the abscissa and ordinate are interchanged, and provides an estimate of the uncertainty of the regression.

the exact magnitude and geographic extent remain uncertain. Mechanisms for climate change over decadal to century time scales may potentially be found in combinations of changing atmospheric trace gas concentrations, North Atlantic Deep Water production, volcanic aerosols, solar radiative output and insolation, and natural variability (Rind \& Overpeck 1993). Shown in Figure 5 are calculations by the GISS GCM of the geographical distribution of surface temperature changes corresponding to a reduction of $0.25 \%$ in solar total irradiance. The global reduction is $0.46^{\circ} \mathrm{C}$. There is significant cooling $\left(0.5^{\circ} \mathrm{C}\right.$ to $1.5^{\circ} \mathrm{C}$ ) at tropical latitudes, but not all regions on the Earth's surface are predicted to cool. Rather, because of differential heating between the land and the oceans, some geographical regions may have been warmer (by as much as $1^{\circ} \mathrm{C}$ ) during the Maunder Minimum.

\section{Discussion}

Our estimates of solar radiative output variations and their impact on the global surface temperature during the Maunder Minimum suggest that improved knowledge of solar forcing will be needed to help reduce uncertainties in interpreting both paleo- and future climate change. Determining whether solar radiative output variations of as much as $0.2 \%-0.3 \%$ are indeed occurring over decadal time scales will require continuous, high-precision solar monitoring for many decades. The first evidence for such long term 


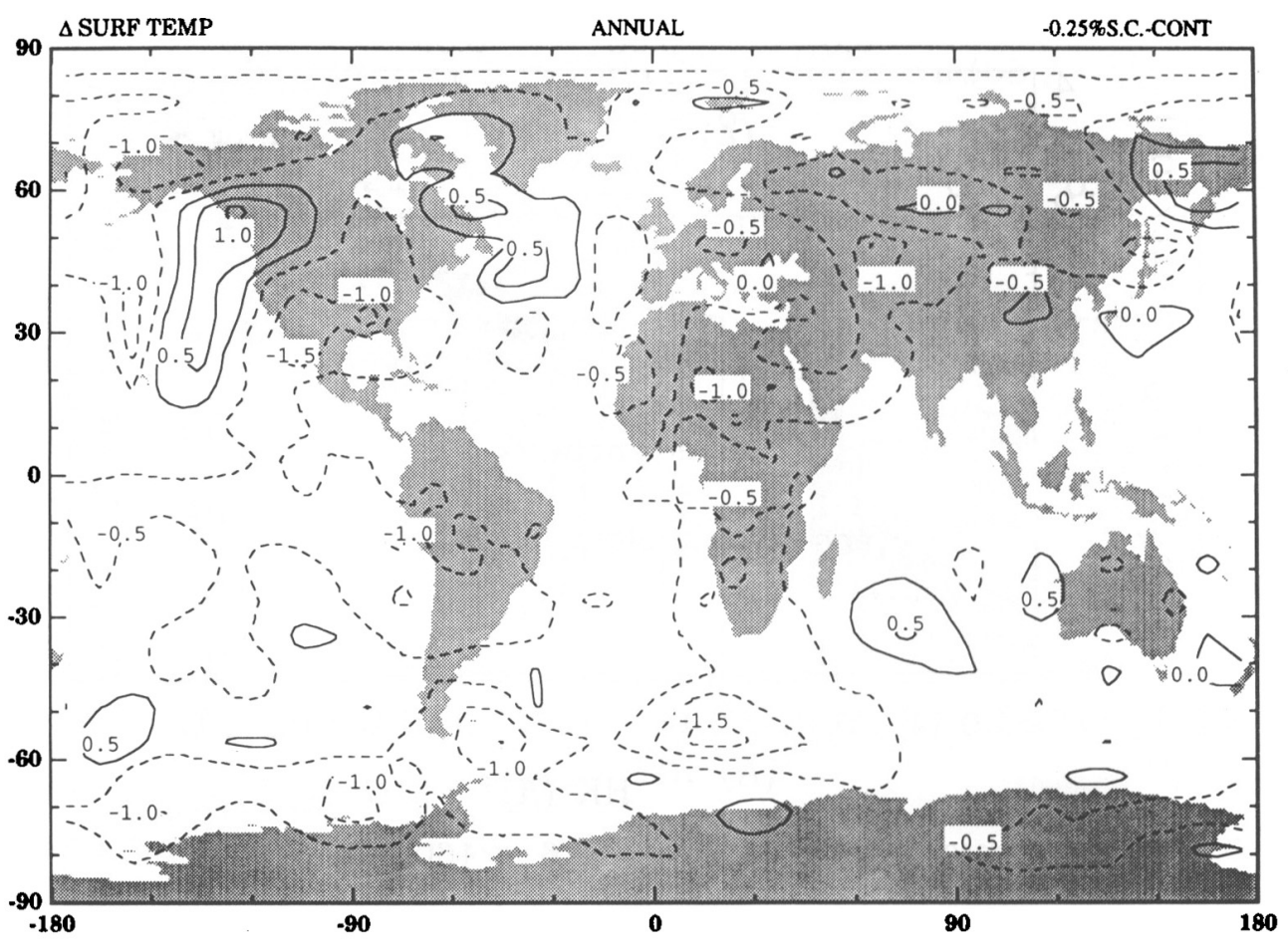

FIgURE 5. Calculations by the GISS GCM of the geographical distribution of the temperature change arising from a spectrally uniform reduction in solar radiative output of $0.25 \%$.

changes might come from comparison of successive minima in the 11-year activity cycle, since the mechanism for the longer term variations, if they are occurring, is expected to affect the overall background level. However, assuming that solar activity is presently at the peak of the Modern Maximum, only very small changes can be expected until a period of rapid change ensues.

Determining the exact nature of the terrestrial response to solar forcing will also require additional effort. The calculations used to produce the results in Figure 5 assumed a flat spectral irradiance reduction. However the solar emission at ultraviolet wavelengths is also expected to be reduced during the Maunder Minimum (Lean et al. 1994, manuscript in preparation). Changes in ultraviolet irradiance at wavelengths shortward of $300 \mathrm{~nm}$ can also impact the surface temperature. To the extent that the irradiance variations occur at wavelengths that are deposited higher in the Earth's atmosphere (rather than penetrating to the Earth's surface), the direct solar forcing of the Maunder Minimum temperature would be reduced. However, atmospheric ozone concentrations would be less because of the diminished ultraviolet radiation, with the corresponding radiative forcing dependent on the altitude profile of the depletion. To investigate this, further studies with the GISS GCM are planned.

Acknowledgments. The solar Ca K data are measured at the National Solar Observatory at Kitt Peak by W. Livingston. 


\section{REFERENCES}

BALIUNAS, S. \& JASTROW, R. 1990 Evidence for long term brightness changes of solar-type stars. Nature 348, 520-523.

Brandt, P. N., Schmidt, W. \& Steinegger, M. 1992 Photometry of sunspots observed at Tenerife. In Proceedings of the Workshop on the Solar Electromagnetic Radiation Study for Solar Cycle 22 (ed. R. F. Donnelly). pp. 130-134. NOAA ERL SEL, Boulder, CO, USA.

DONNELLY, R. F. 1988 Uniformity in solar flux variations important to the stratosphere. Annales Geophys. 6, 417-424.

EdDy, J. A. 1976 The Maunder Minimum. Science 192, 1189-1202.

Foukal, P. 1981 Sunspots and changes in the global output of the Sun. In Physics of Sunspots (ed. L. E. Cram \& J. H. Thomas). pp. 391. Sacramento Peak Observatory, Sunspot, NM, USA.

FoukaL, P. \& LEAN, J. 1988 Magnetic modulation of solar luminosity by photospheric activity. Astrophys. J. 328, 347-357.

Foukal, P. \& LEAN, J. 1990 An empirical model of total solar irradiance variation between 1874 and 1988. Science 247, 505-604.

FröHLICH, C. 1994 Irradiance observations of the Sun. In The Sun as a Variable Star: Solar and Stellar Irradiance Variations (ed. J.M. Pap, C. Fröhlich, H.S. Hudson \& S.K. Solanki). Cambridge University Press, in press.

Hoyt, D. V., KYLE, H.L., Hickey, J. \& MaschHoff, R. 1992 The Nimbus 7 total solar irradiance: A new algorithm for its derivation. J. Geophys. Res. 97, 51-63.

Kuhn, J. R. \& Libbrecht, K. G. 1991 Nonfacular solar luminosity variations. Astrophys. J., L35-L37.

LEAN, J. 1991 Variations in the Sun's radiative output. Rev. Geophys. 29, 505-535.

Lean, J., Skumanich, A. \& White, O. 1992 Estimating the Sun's radiative output during the Maunder Minimum. Geophys. Res. Lett. 19, 1591-1594.

Livingston, W. C., Wallace, L. \& White, O. R. 1988 Spectrum line intensity as a surrogate for solar irradiance variations. Science, 240, 1765-1767.

LOCKWOOD, W. 1993 Irradiance variations of stars. In The Sun as a Variable Star: Solar and Stellar Irradiance Variations (ed. J.M. Pap, C. Fröhlich, H.S. Hudson \& S.K. Solanki). Cambridge University Press, in press.

Mchargue, L. R. \& Damon, P.E. 1991 The global Beryllium 10 cycle. Rev. Geophys. 29, 141-158.

RIND, D. \& OVERPECK, J. 1993 Hypothesized causes of decadal-to-century climate variability: climage model results. Quat. Sci. Rev., in press.

Rottman, G. J. 1988 Observations of solar UV and EUV variability. Adv. Space Res. (7)53(7)66.

Skumanich, A., Smythe, C. \& Frazier, E. N. 1975 On the statistical description of inhomogeneities in the quiet solar atmosphere. I. Linear regression analysis of chromospheric variability in the Calcium K line. Astrophys. J. 200, 747-764.

Skumanich, A., Lean, J. L., White, O. R. \& Livingston, W. C. 1984 The Sun as a star: Three-component analysis of chromospheric variability in the Calcium $\mathrm{K}$ line. Astrophys. J. 282, 776-783.

White, O. R., Rottman, G. J. \& Livingston, W. C. 1990 Estimation of the solar Lyman alpha flux from ground based measurements of the CaII K line. Geophys. Res. Lett., 17, 575-578.

White, O. R., Skumanich, A., Lean, J., Livingston W. C. \& Keil, S. L. 1992 The Sun in a non-cycling state. Publ. Astronom. Soc. Pac. 104, 1139-1143.

Willson, R. C. \& Hudson, H. S. 1991 The Sun's luminosity over a complete solar cycle. Nature 351, 42-44. 